\title{
IDENTIFICAÇÃO DAS VARIAÇÕES DE SEGREGAÇÕES EM BARRAS DE AÇO ABNT NM 87 GRAU 1045 ORIUNDAS DO PROCESSO DE TREFILAÇÃO COMBINADA*
}

\author{
Rachel Ribeiro Possamai ${ }^{1}$ \\ Vinicius Waechter Dias ${ }^{2}$ \\ Rodrigo Afonso Hatwig ${ }^{3}$ \\ Juliana Zottis ${ }^{4}$ \\ Alexandre da Silva Rocha ${ }^{5}$
}

\section{Resumo}

No processo de fabricação de componentes mecânicos existem vários fatores que contribuem para gerar distorções de forma após o tratamento térmico, sendo um deles o desalinhamento na distribuição da zona de segregação. Este artigo tem como objetivo caracterizar as segregações em barras de aço ABNT NM 87 Grau 1045, assim como determinar sua variação dentro de um mesmo lote. Para tanto, foram retiradas amostras de diferentes posições de uma rota de trefilação combinada, nas quais foram realizadas análises químicas e microestruturais, com o intuito de caracterizar as zonas de segregação ao longo de um fio-máquina durante o processo de trefilação. Os resultados das análises químicas mostram uma maior concentração de carbono na zona de segregação, com um pico de 0,7\% em massa. A análise microestrutural apresenta uma maior concentração de perlita próximo ao centro. As análises macrográficas demonstram que há uma variação na segregação dentro do mesmo lote, tanto em posição quanto em concentração.

Palavras-chave: Trefilação combinada; Aço ABNT NM 87 Grau 1045; Segregação de carbono.

\section{IDENTIFICATION OF CHANGES IN SEGREGATIONS IN ABNT 1045 STEEL BARS FROM THE COMBINED DRAWING PROCESS}

\section{Abstract}

In the manufacturing of mechanical components there are several factors that contribute to generate distortions after heat treatment. One of them is the misalignment in the distribution of the segregation zone. This article aims to characterize the segregations in ABNT 1045 steel drawn bars, as well as to predict their variation within the same heat (wire-rod). For this purpose, samples were taken from different locations of a combined drawing process route. Chemical, macrostructural and microstructural analysis were done to characterize the segregation zone along the wire-rod. The chemical composition showed higher concentration of carbon in the segregation zone with a peak of $0.7 \%$ in mass. A higher concentration of perlite near the center was observed in the microstructural analysis. The macrographic analysis showed that segregations varies in the same batch, both in position and in concentration.

Keywords: Drawing Chain; ABNT 1045 steel; Carbon segregation.

1 Graduanda em Eng. Metalúrgica, LdTM/GES, Universidade Federal do Rio Grande do Sul, Porto Alegre, RS, Brasil.

MSc. Eng. Metalúrgico, LdTM/GES, UFRGS, Porto Alegre, RS, Brasil.

MSc. Eng. Metalúrgico, LdTM/GES, UFRGS, Porto Alegre, RS, Brasil.

MSc. Eng. Mecânica, LdTM/GES, UFRGS, Porto Alegre, RS, Brasil.

Dr. Prof. MSc Eng. Mecânico, LdTM, UFRGS, Porto Alegre, RS, Brasil. 


\section{INTRODUÇÃO}

A trefilação combinada é um processo de conformação mecânica que tem como objetivo a melhoria das propriedades mecânicas do material [1]. Este processo se dá pelas seguintes etapas: inicia-se no desbobinamento do fio-máquina, para então passar para o processo de pré-endireitamento horizontal, seguido do préendireitamento vertical. O pré-endireitamento é realizado por múltiplos rolos e tem como finalidade deixar o fio-máquina o mais retilíneo possível. Em seguida vem a etapa do jateamento, que é a decapagem mecânica das barras por jateamento de granalha, responsável pela remoção da carepa do material e preparação da superfície para a etapa de trefilação.

O processo de trefilação pode ser definido como um processo de manufatura por deformação plástica, onde o fio-máquina é tracionado passando através de uma ferramenta cônica, causando uma redução da área da seção transversal e aumentando o comprimento total [2]. Em seguida é realizado o corte do material em barras de 6 metros, para então seguir para o PERC (processo de Polimento e Endireitamento por Rolos Cruzados côncavos e convexos), etapa que proporciona o acabamento das barras. Todas as etapas da trefilação combinada são dispostas na Figura 1.

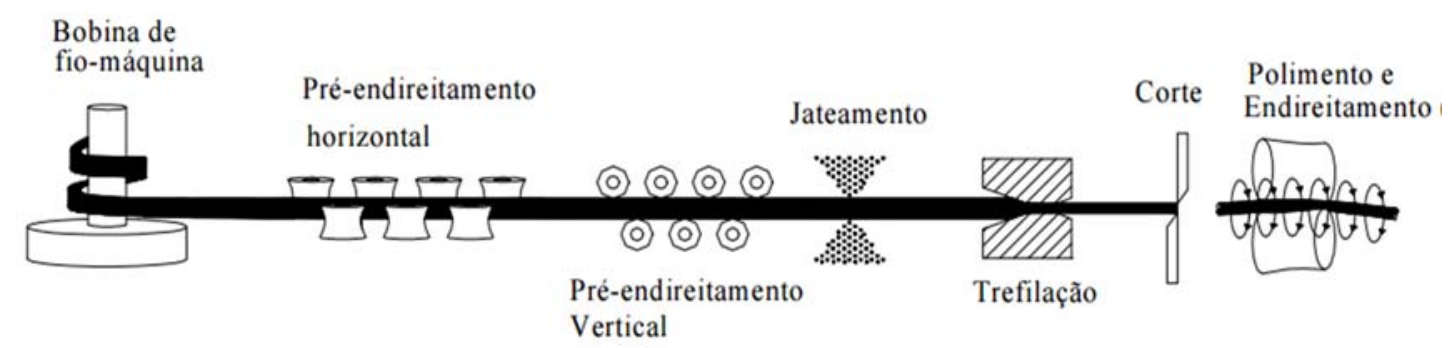

Figura 1. Etapas do processo de trefilação combinada. Fonte: Nunes, 2012

No processo de trefilação existem vários fatores que podem gerar distorções de forma após o tratamento térmico, sendo alguns deles a distribuição das tensões residuais e o desalinhamento na distribuição da zona de segregação [3]. As segregações são geradas durante o processo de solidificação do material, podendo ser alteradas e redistribuídas durante o processo de fabricação [4].

Esta pesquisa faz parte do projeto "Investigation and improvement of a manufacturing process chain from cold drawing processes to induction hardening" em parceria com o programa BRAGECRIM (Brazilian German Collaborative Research Initiative in Manufacturing Tecnology), que visa estudar os fenômenos ocorridos durante o processo de fabricação de eixos automotivos através da "engenharia de distorção". O estudo deste artigo se aplica à fabricação das barras, tendo como objetivo caracterizar as segregações em barras de aço ABNT 1045, assim como determinar sua variação dentro de um mesmo lote do material.

\section{MATERIAIS E MÉTODOS}

Para a realização das análises, foram retiradas pequenas barras de aço ABNT NM 87 Grau 1045 de diferentes posições de um mesmo lote de material em uma rota de trefilação combinada (Figura 1), sendo elas: uma amostra do fio-máquina, duas amostras do pré-endireitamento (horizontal e vertical, respectivamente), uma do jateamento, uma da trefila e uma amostra do PERC, totalizando seis (6) amostras. 
Os cortes das amostras foram realizados utilizando um equipamento de corte com serra fita, no tamanho de $10 \mathrm{~mm}$ de comprimento, retiradas da ponta inicial de cada barra, conforme a Figura 2.

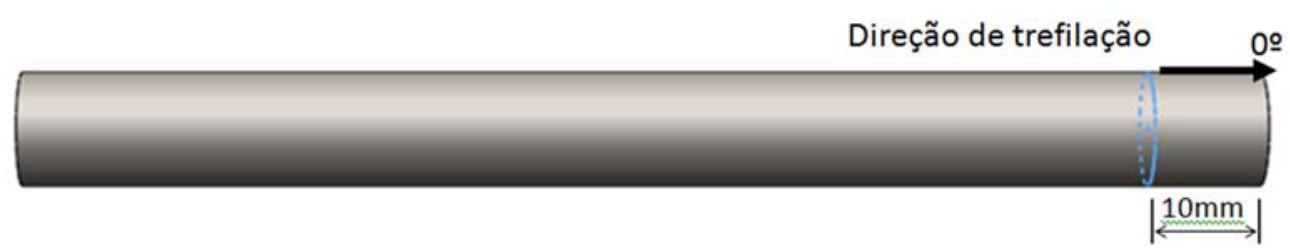

Figura 2. Representação do corte das amostras.

Para a retirada de amostras foi determinado um sistema de orientação guiada que consiste na marcação da direção de trefilação e a definição de um ponto de referência denominado posição $0^{\circ}$ relacionado à posição superior da fieira, ilustrado na Figura 3. Tal posição serve como referencial para a comparação entre as amostras de diferentes posições do fio-máquina [5]. Tendo isso em vista, após a realização dos respectivos cortes foi realizada a marcação desta posição nas amostras com um marcador elétrico.

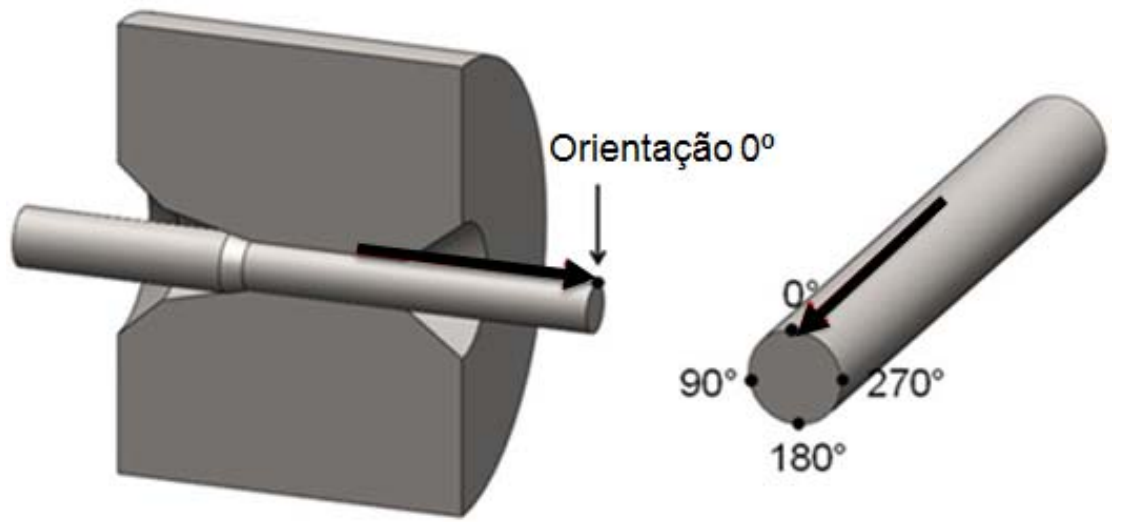

Figura 3. Representação do corte das amostras [6]

Na preparação metalográfica as amostras passam por uma série de lixas, sendo elas de granulometria entre 100 e 1200, do acabamento mais grosseiro ao mais refinado, respectivamente. O lixamento deve ser realizado com água corrente, a fim de descartar o material originado do desgaste tanto do próprio material quanto da lixa. As amostras devem ser giradas $90^{\circ}$ a cada troca de lixa a fim de que todos os riscos da lixa anterior contidos nas amostras sejam eliminados. É realizada a troca de lixa assim que todas as linhas visíveis estejam na mesma direção. Em seguida é feito o polimento da amostra com pasta de diamante. Tal procedimento tem como objetivo fazer com que as amostras fiquem com uma superfície lisa e espelhada para então ser realizado o ataque químico da amostra. Para as análises microscópicas, foi utilizado Nital $2 \%$, enquanto que para a análise macrográfica utilizou-se Nital $5 \%$. O ataque químico é realizado para revelar as diferentes fases da microestrutura contida no metal, assim como revelar suas segregações.

Foi realizada análise química (espectroscopia por emissão ótica) e EPMA (Electron Probe Micro-Analysis) de três amostras (duas necessárias para a análise química e uma para o EPMA), coletadas após a etapa de trefilação, a fim de que se pudesse fazer uma melhor comparação entre as zonas segregadas com o restante da amostra. A análise química foi realizada com o intuito de verificar se há variação dos 
componentes químicos entre a zona de segregação com o restante da amostra e o EPMA apresenta um estudo mais aprofundado da quantidade de carbono contida no material [7]. Além disto, foram realizadas análises através de macrografia e microscopia ótica, utilizando o microscópio Olympus GX51, com o intuito de analisar a variação microestrutural entre a região segregada e a região fora da segregação.

\section{RESULTADOS E DISCUSSÃO}

A variação local da composição química do material e a sua microestrutura foram primeiramente analisadas via espectroscopia por emissão ótica e EPMA, para só então ser realizada a análise microscópica e macroscópica. A partir das análises químicas, pode ser observada uma maior quantidade de carbono no ponto central da amostra (1) em comparação aos pontos periféricos (2 a 5). A Figura 4 mostra o local da amostra onde foram realizadas as análises e a Tabela 1 mostra o resultado obtido comparado à composição nominal [8].
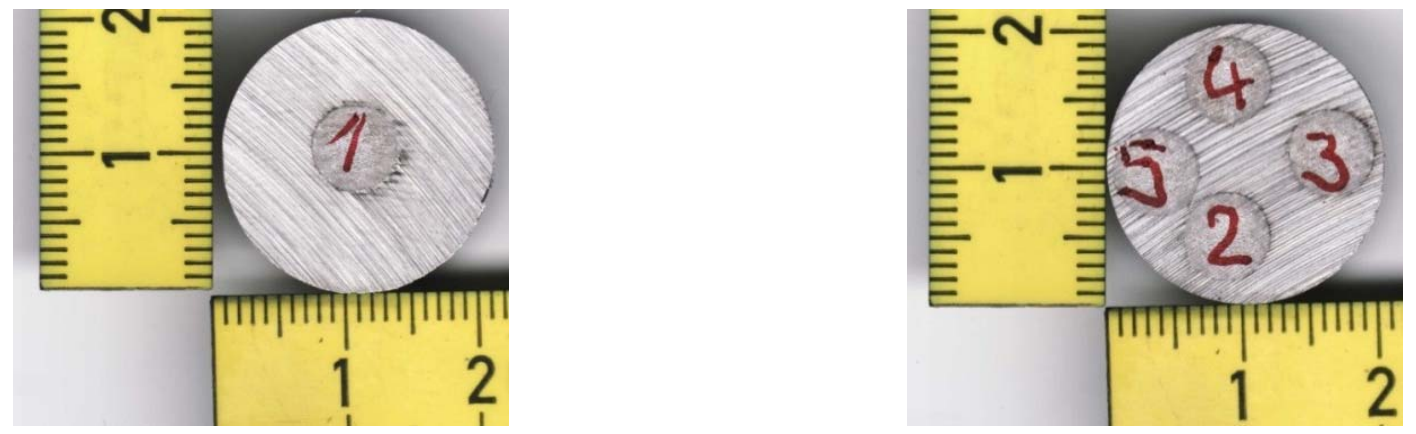

Figura 4. Pontos utilizados para a análise química.

Tabela 1. Composição química (\% em massa) para os diferentes pontos medidos

\begin{tabular}{cccccc}
\hline & $\mathbf{C}$ & $\mathbf{M n}$ & $\mathbf{S i}$ & $\mathbf{P}$ & $\mathbf{S}$ \\
\hline \hline $\mathbf{1}$ & 0,49 & 0,877 & 0,237 & 0,025 & 0,035 \\
\hline $\mathbf{2}$ & 0,47 & 0,871 & 0,233 & 0,025 & 0,036 \\
\hline $\mathbf{3}$ & 0,47 & 0,868 & 0,236 & 0,025 & 0,037 \\
\hline $\mathbf{4}$ & 0,47 & 0,869 & 0,235 & 0,024 & 0,034 \\
\hline $\mathbf{5}$ & 0,47 & 0,870 & 0,235 & 0,024 & 0,034 \\
\hline Nominal & $0,42-0,5$ & $0,6-0,9$ & N.I. & $<0,04$ & $<0,05$ \\
\hline
\end{tabular}

Esta mesma segregação central analisada quimicamente pode ser observada macroscopicamente em sua seção longitudinal como uma linha mais escurecida, apresentando variações ao longo da seção axial, conforme a Figura 5a. A Figura 5b mostra o teor de carbono localizado ao longo do diâmetro do eixo de uma amostra após a trefila. A imagem acima do gráfico representa a microestrutura da área analisada com $20 \mu \mathrm{m}$ de amplitude, no qual a concentração de carbono detectada a partir de uma rede de 1000 pontos ao longo do diâmetro e 200 pontos de largura resultou na distribuição de carbono ao longo do diâmetro representado na Figura $5 b$. 


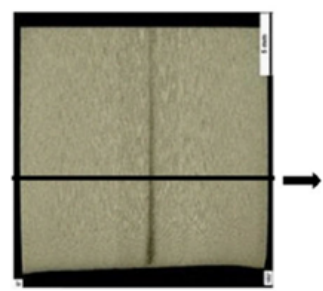

(a)

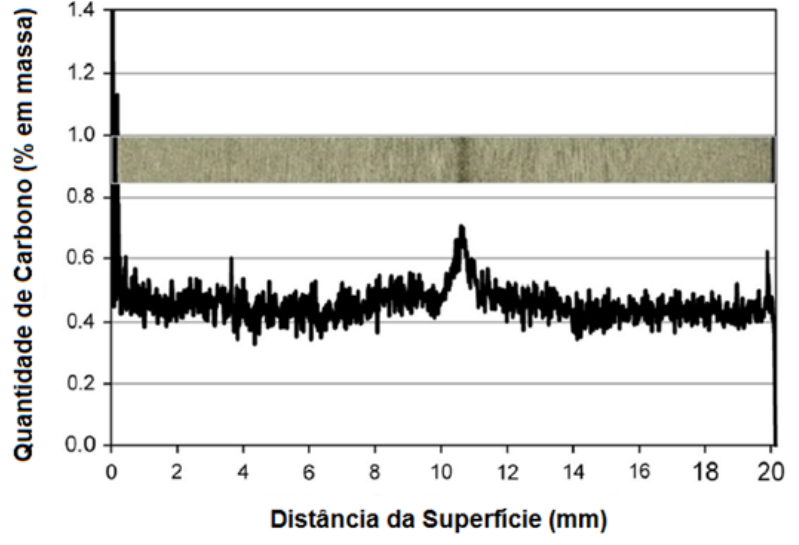

(b)

Figura 5. Macroestrutura da seção longitudinal (a); (b) distribuição da quantidade de carbono (linha) analisado pelo EPMA ao longo do diâmetro da amostra com $20 \mu \mathrm{m}$ de largura marcado com a linha em (a).

Observou-se também um aumento nos teores de carbono e manganês em relação aos valores médios para este aço: na área central o valor máximo atingido de carbono foi de $0,7 \%$ em massa, e o valor máximo atingido de manganês foi de $1,4 \%$ em massa na área mais escura da microestrutura. Também pode ser observado que a posição da segregação é excêntrica por cerca de 0,6 mm.

A microestrutura da amostra é caracterizada por uma concentração de perlita em sua zona de segregação, assim como ilustrado na Figura 6. Isto ocorre devido à macrosegregações de elementos de liga durante o processo de solidificação. $O$ restante da amostra apresenta uma maior quantidade de ferrita.

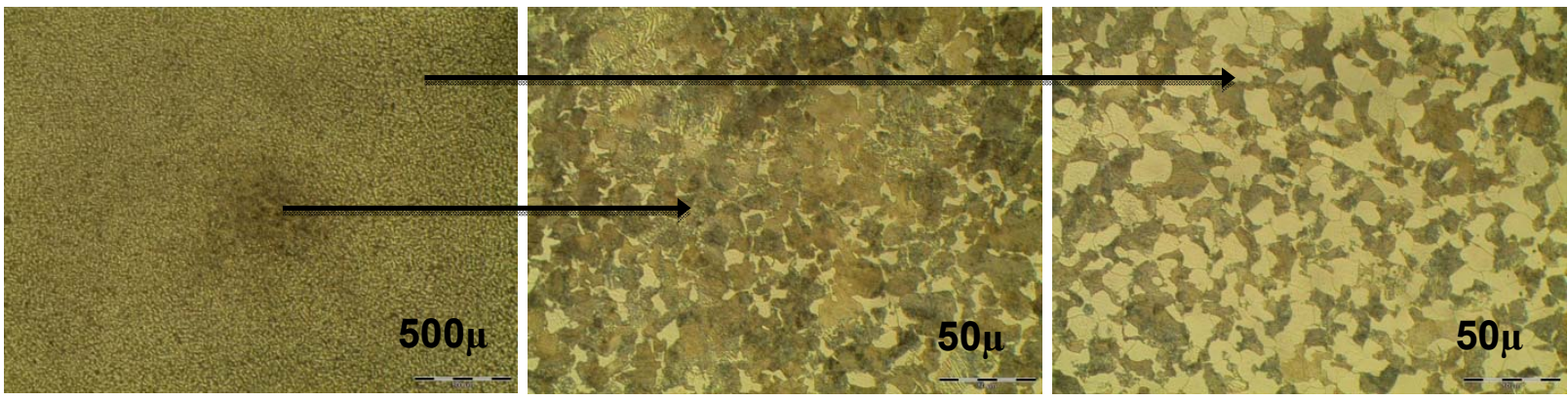

Figura 6. Microscopia da parte inicial de uma amostra do PERC, em aumentos de 5x e 50x, respectivamente.

A partir das análises químicas e microscópicas foi confirmada a existência de uma zona de segregação no material. Foram então realizadas análises macrográficas nas amostras, apresentadas nas Figuras 7 a 12. O ângulo $0^{\circ}$ indica a direção de trefilação. A amostra do fio-máquina (Figura 7) possui uma região de segregação bastante dispersa, o que a torna menos visível. 


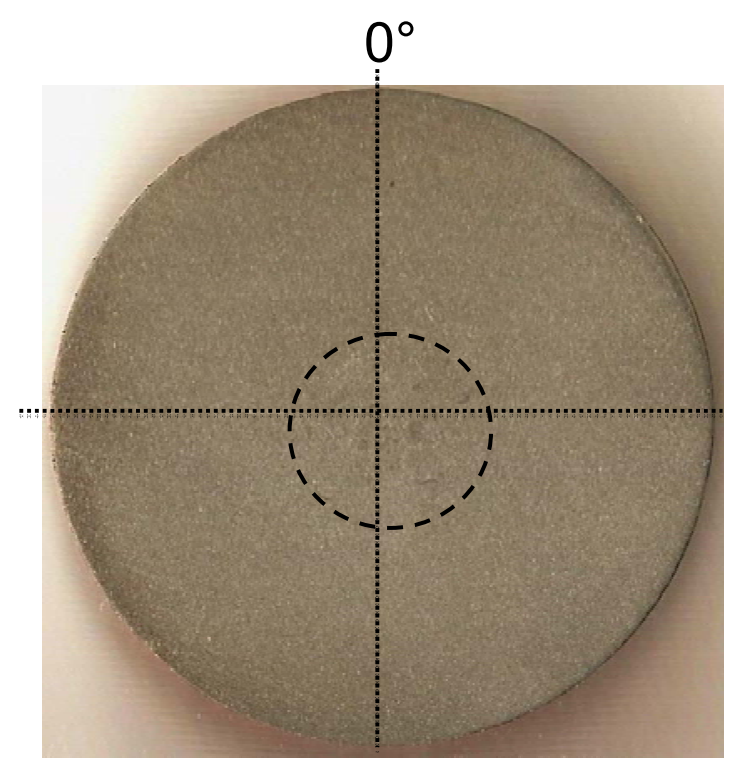

Figura 7. Amostra do fio-máquina.

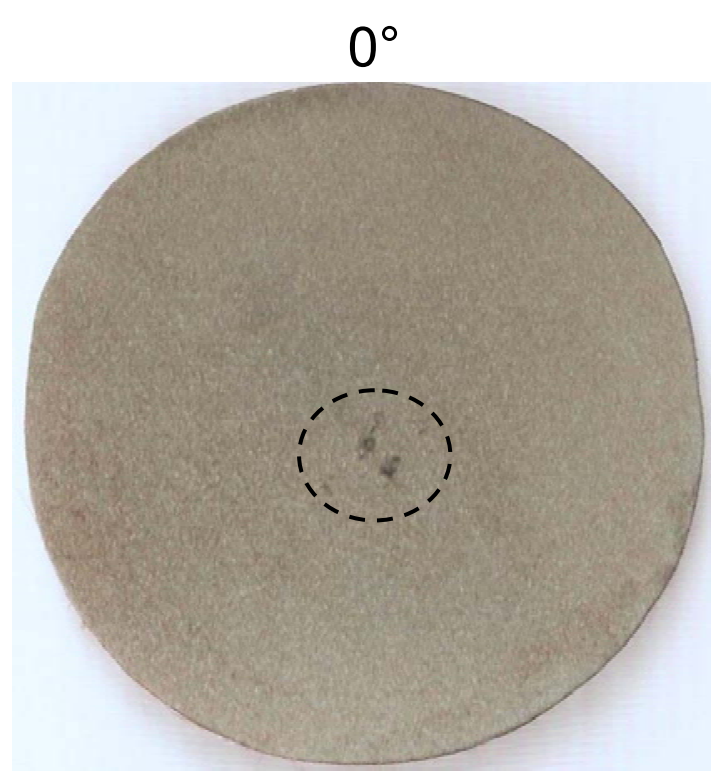

Figura 9. Amostra do pré-endireitamento vertical.

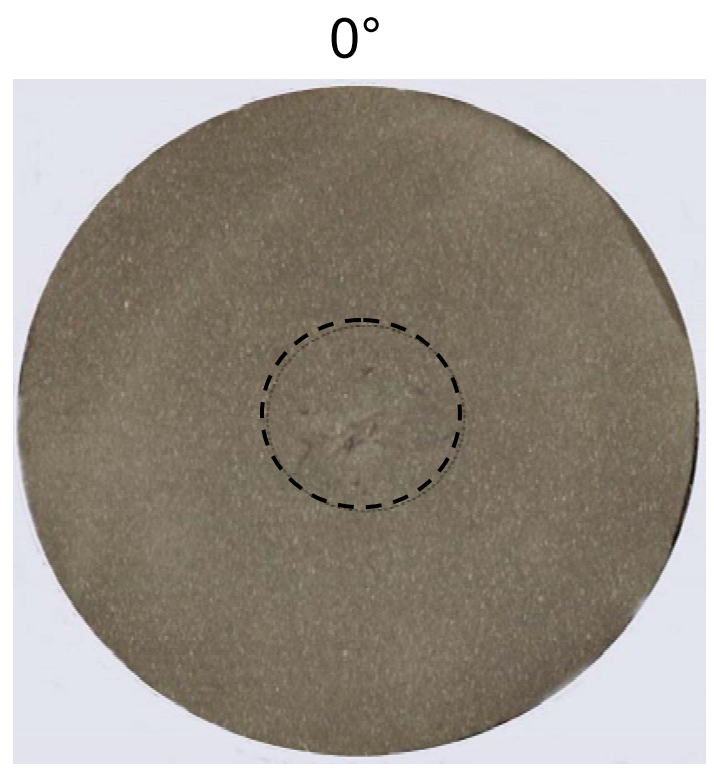

Figura 8. Amostra do pré-endireitamento horizontal.

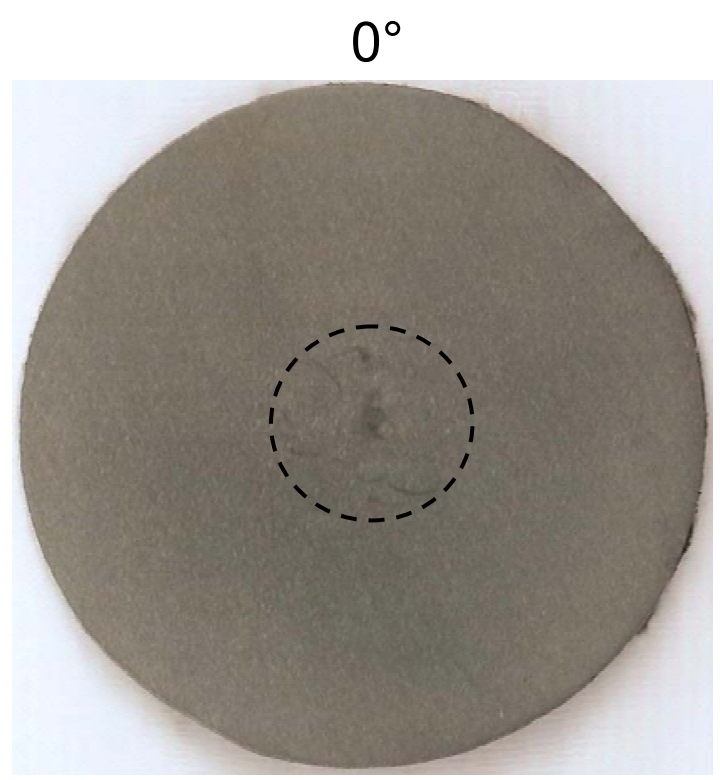

Figura 10. Amostra do jateamento. 


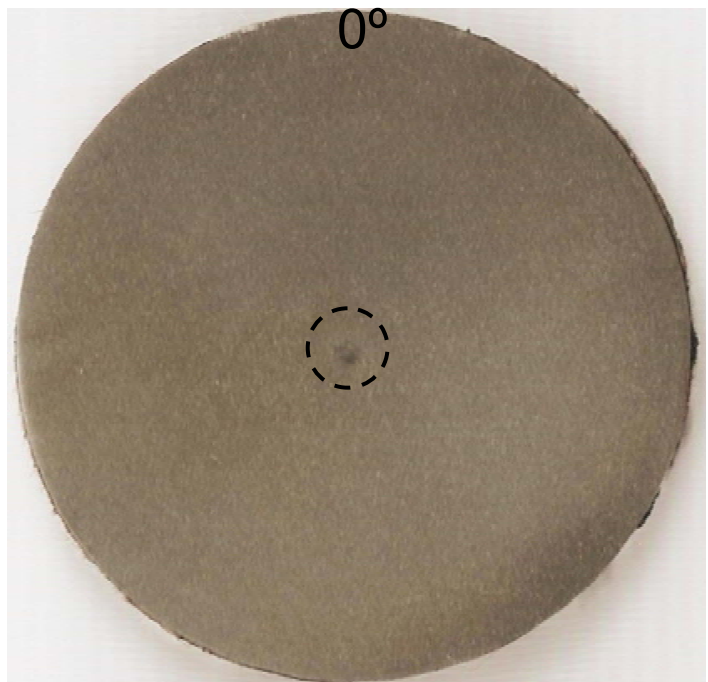

Figura 11. Amostra da trefila.

Figura 12. Amostra do PERC.

Ambas as amostras do pré-endireitamento mostram segregações descentralizadas, sendo a amostra do pré-endireitamento horizontal (Figura 8) bem semelhante à amostra do fio-máquina. Já a amostra do pré-endireitamento vertical (Figura 9) mostra uma maior concentração da zona de segregação.

A amostra retirada da etapa do jateamento (Figura 10) apresenta segregação aparente à amostra do fio-máquina, porém mais concentrada. A partir da amostra da trefila (Figura 11) é verificado que há apenas um ponto de segregação próximo ao centro. Desta maneira, pode-se concluir que a zona de segregação do aço ABNT 1045 não é homogênea e varia tanto em forma quanto em posição dentro de um mesmo lote de fio-máquina.

\section{CONCLUSÃO}

Através das análises químicas foi comprovado o maior do percentual de carbono e manganês próximo ao núcleo da amostra, em comparação ao restante do material e à composição nominal.

Com as análises microscópicas detectou-se uma alta concentração de estrutura perlítica nas regiões de segregações.

Através das análises macrográficas foi possível verificar que as segregações variam tanto em posição quanto em concentração em diferentes partes do mesmo fiomáquina.

Portanto, foi possível caracterizar as segregações do lote analisado determinando que existem variações da posição e quantidade de carbono. Estes resultados servirão de base para maiores investigações sobre a sua influência no aparecimento de distorções do produto final.

\section{Agradecimentos}

Os autores a! ıesquisa, realizada entre o período de 01/07/2015 a 30/04/2016, número do processo 115722/2015-7. 


\section{REFERÊNCIAS}

1 FERLAUTO, E.M. Heterogeneidades mecânicas e microestruturais durante o processo de trefilação combinada do aço SAE 1045. Dissertação para obtenção do título de mestre em engenharia. Universidade Federal do Rio Grande do Sul, PPGE3M. 2011.

2 DIETER, G.E.; HOWARD, A.K.; SEMIATIN, S.L. Handbook of workability and process design. ASM International, Ohio, 2003, p-278.

3 NUNES, R. M. Estudo de distorção de barras cilíndricas de Aço ABNT 1045, em uma rota de fabricação envolvendo trefilação combinada e têmpera por indução.

Dissertação para obtenção de título de doutor em engenharia. Universidade Federal do Rio Grande do Sul, PPGE3M. 2012.

4 SIMSIR, C.; GÜR, C.H. 2008. A new framework for simulation of heat treatments. IDE 2008, Bremen, Germany, September 17th - 19th. 471-480.

5 THOBE K.D., KLEIN, D., SEIFERT, M., WUEST, T. 2012. Approaches to describe distortion along process chains. Materialwissenschaft Und Werkstofftechnick, 43(1-2), pp. 178-185.

6 DIAS, V.W. Influência dos procedimentos de corte sobre as distribuições de tensões residuais em barras redondas trefiladas de aço ABNT 1045. Dissertação para obtenção do título de mestre em engenharia. Universidade Federal do Rio Grande do Sul, PPGE3M. 2013.

7 DONG J., EPP J., ROCHA A.S., NUNES R.M., ZOCH. H.W. Investigation of the influence factors on distortion in induction-hardened steel shafts manufactured from cold-drawn rod. The Minerals, Metals \& Materials Society and ASM International, Volume 47a, pp. 877-888. 2016.

8 MatWeb - Metal Property Data [acesso em 12 de abril de 2016]. Disponível em: http://www.matweb.com/search/datasheet_print.aspx?matguid=193434cf42e343fab880 e1dabdb143ba 